\title{
On the Acoustics of a Circulation Control Airfoil
}

\author{
R. Reger ${ }^{a, *}$, A. Nickels ${ }^{b}$, L. Ukeiley ${ }^{b}$, L. N. Cattafesta $^{a}$ \\ ${ }^{a}$ Department of Mechanical Engineering, Florida Center for Advanced Aero-Propulsion, \\ Florida State University, Tallahassee, FL 32310, USA \\ ${ }^{b}$ Department of Mechanical and Aerospace Engineering, \\ University of Florida, Gainesville, FL 32611, USA
}

\section{Abstract}

A two-dimensional elliptical circulation control airfoil model is studied in the Florida State Aeroacoustic Tunnel. Far-field acoustics are obtained via a 55 microphone phased array. Single microphone spectra are also obtained, and it is shown that background noise is significant. In order to circumvent this problem, beamforming is employed. The primary sources of background noise are from the tunnel collector and jet/sidewall interaction. The deconvolution approach to mapping acoustic sources (DAMAS) is employed to remove the effects of the array point spread function. Spectra are acquired by integrating the DAMAS result over the source region. The resulting DAMAS spectral levels are significantly below single microphone levels. Although the DAMAS levels are reduced from those of a single microphone or delay and sum beamforming (DAS), they are still above those of a NACA 0012, estimated using NAFNoise, at the same geometric and free-stream conditions. A scaling analysis is performed on the processed array data. With a constant free-stream velocity and a varying jet velocity the data scale as jet Mach number to the 6 th power. If the momentum coefficient is held constant and the free-stream velocity is varied the data scale as free-stream Mach number to the 7 th power.

\section{Introduction}

Traditionally, lift is proportional to the square of the forward velocity of a body. This means that vehicles moving at very low speeds, such as submarines or aircraft during approach, have drastically reduced maneuverability, making them more difficult to control. Circulation control (CC) provides a mechanism for increased lift and maneuverability even at low forward speeds. On air- and hydro-foils, CC generally provides these advantages by issuing a high speed jet from a spanwise slot, tangential to a curved trailing edge. The jet entrains low-momentum fluid, and the flow stays attached longer due to the Coanda effect. This delays separation and increases circulation by shifting the stagnation point, which results in augmented lift [1]. Due to this lift increase, CC could allow for reduced mechanical complexity on aircraft and underwater vehicles in the event that it replaces current mechanical systems that change the angle of attack. The fluid dynamic mechanisms and benefits of CC have been studied extensively and are well understood [2]. Though the advantages gained from CC are significant, it comes with drawbacks [3].

One of the issues presented by $\mathrm{CC}$ is the production of significant levels of undesirable acoustic noise. This additional noise is detrimental to its implementation both in air and underwater. In air, strict requirements are placed on acoustic levels for the areas surrounding airports. Underwater, $\mathrm{CC}$ noise reduces stealth capabilities and sonar system reliability and therefore lifespan of the vehicle. Compared to the copious number of studies on CC fluid dynamics, there is a relative scarcity of work regarding CC acoustics.

The first study on CC acoustics was performed by Williams and Cheeseman [4] who suggested ten primary sources of rotor-craft CC noise. Later Salikuddin et al. [5] performed experiments varying jet speed and slot height, finding that noise levels increased as either parameter increased. Howe [6] then

\footnotetext{
*Corresponding author, robert.w.reger@gmail.com
}

Preprint submitted to Elsevier

October 7, 2016 

created an analytic model to predict $\mathrm{CC}$ acoustic radiation. He suggested that three CC sources dominate: 1.) Curvature noise generated by free-stream turbulence interacting with the trailing edge dominates at low frequencies, 2.) Passive-slot noise generated by free-stream turbulence interacting with the slot lip dominates at middle frequencies, and 3.) Jet-slot noise generated from turbulence in the jet interacting with the slot lip dominates at high frequencies. In recent years, Shannon and Morris [7], Morris et al. [8], Wetzel [2], and Wetzel et al. [9] performed experiments to determine CC noise. Morris et al. [8], Wetzel [2] and Wetzel et al. [9] show that the model of Howe considerably underestimates the CC noise.

These recent studies make use of phased acoustic arrays for a limited subset of cases but focus primarily on spectral results from single microphones. Phased acoustic arrays employ a large number of microphones at known spatial locations and are processed using various beamforming algorithms [10]. Using knowledge of the source locations relative to objects of interest, one can integrate over areas relevant to the model noise effectively eliminating background noise [11]. This offers a significant advantage over single microphone measurements, which can be envisioned as simply integrating over the entire field (including the external noise). In the current study, it is shown that background and external noise are significant and tend to saturate the single microphone results, especially at low jet velocities. In order to remove these effective background noise sources, beamforming and selective integration regions are employed.

While previous studies provide beammaps, the results are limited to those from delay-and-sum (DAS) beamforming and only for a limited number of cases. In DAS beamforming the source field is convolved with the array geometry for an ideal point source, or the array's point spread function (PSF) [12]. The PSF represents the ideal point source that the array geometry can provide at each location in space. In the best case, the PSF causes a point source to appear spread spatially over several locations, especially at low frequencies while in the worst case, it generates erroneous sources (known as sidelobes) causing imprecise source locations and magnitudes [12]. The current study performs both conventional (DAS) beamforming as well as a deconvolution approach for the mapping of acoustic sources (DAMAS) [13] to remove the effects of array geometry and to obtain more accurate estimates of the source locations and spectra.

The purpose of the present study is to gain a better understanding of noise generated by circulation control via (1) isolation of the sources of interest from effective background noise, (2) comparison to traditional lifting appendages, and (3) presentation of an acoustic scaling analysis. The following section includes an overview of the experimental setup, including a discussion of the beamforming methods employed. Then, results are provided regarding single microphone, DAS, and DAMAS, followed by a comparison of CC noise with estimated NACA 0012 noise. Finally, a scaling analysis of the source levels is provided, followed by the conclusions. The results are expected to provide some guidance for development of advanced noise prediction methods involving circulation control.

\section{Experimental Setup}

\subsection{Model Overview}

The circulation control airfoil employed in these experiments is the one described by Wetzel [2], whose design is based on the hydrofoil design of Rogers and Donnelly [14], and is briefly discussed here for completeness. The airfoil is two-dimensional and dual-slotted to allow for both upper and lower slot blowing; however, only the upper slot is used in this work. The model is elliptical with a $20 \%$ thickness-to-chord ratio, no camber and a circular trailing edge, detailed in Figure 1. The airfoil has a chord of $0.521 \mathrm{~m}$ and a span of $0.914 \mathrm{~m}$. The trailing edge geometry is shown in Figure 2 where $t_{l}$ indicates the lip thickness.

The interior of the model acts as a plenum and is split in order to separate the supply for the upper and lower blowing slots. The plenum is supplied from a dried, filtered, compressed air source external to the wind tunnel facility. In order to set the height of each slot, eight sets of push-pull screws are placed on each side. All cases discussed have one slot height set to $1 \mathrm{~mm}(h / c=0.0019)$ while the other slot is closed and sealed with tape. The height of the slot is set by a shim material. Several measures are taken to reduce internal noise. First, the plenum is supplied from both spanwise ends of the model. This decreases plenum noise by reducing the flow rate required by either side, thereby reducing the velocity of the flow entering the model and, by extension, the internal noise. This also helps to ensure flow uniformity and minimize flow 


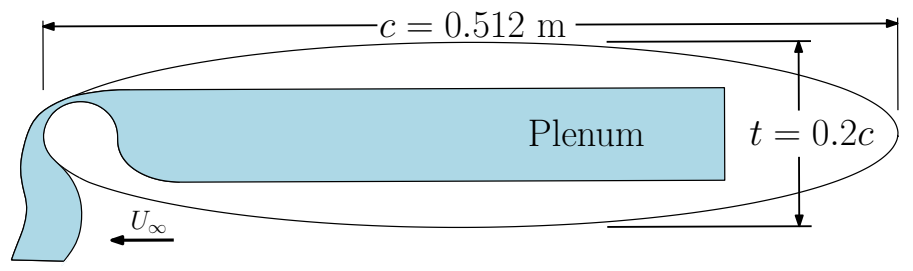

Figure 1: Single side blowing circulation control airfoil with geometric dimensions. (Adapted from [2])

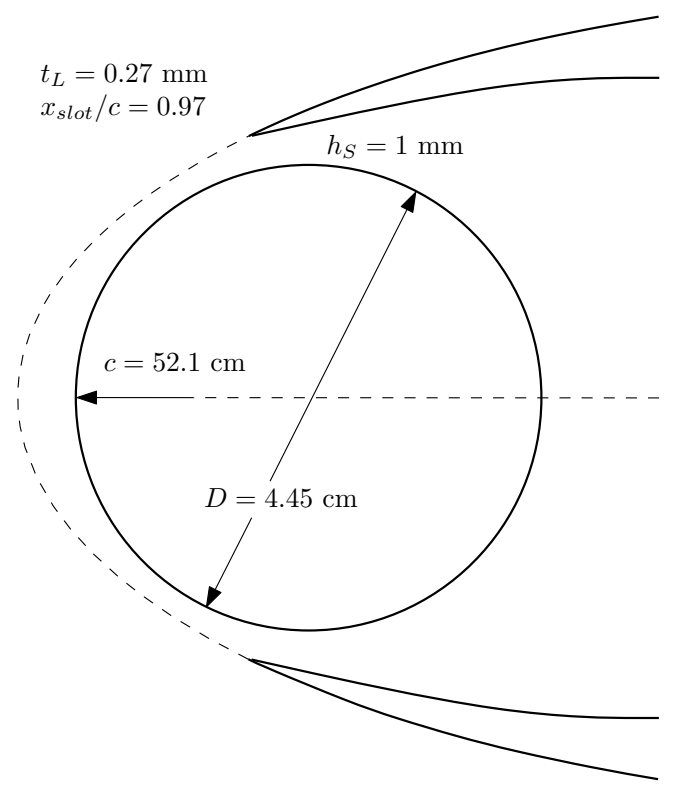

Figure 2: Trailing edge geometry. (Adapted from [2])

speed within the plenum. Next, the flow encounters Poly-fil polyester filling to attenuate the noise from the incoming flow. At the mid-chord, strips of ERG Duocel Aluminum Foam (40, 20, and 10 pores per inch) are installed to reduce noise. The aluminum foam also helps to straighten the flow within the plenum. Finally, sheets of $2 \mathrm{~mm}$ thick foam are installed along the plenum walls to further decrease internal noise.

\subsection{Florida State Aeroacoustic Tunnel}

The Florida State Aeroacoustic Tunnel (FSAT) is an open-return wind tunnel with an open-jet test section. The wind tunnel is housed in an ISO 3745-certified $250 \mathrm{~Hz}$ anechoic room used to simulate an acoustic free-field. Air is pulled through the $0.91 \mathrm{~m}$ tall by $1.22 \mathrm{~m}$ wide by $3.05 \mathrm{~m}$ long test section by a 450-hp centrifugal blower connected to a jet collector via an acoustically lined diffuser. The tunnel is capable of producing free-stream velocities from 5 to $75 \mathrm{~m} / \mathrm{s}$ with turbulence intensity levels below $0.12 \%$ and non-uniformity below $0.1 \%$ [15]. The anechoic room which houses the test section has dimensions of $2.7 \mathrm{~m}$ by $4.5 \mathrm{~m}$ by $4.8 \mathrm{~m}$ (height by width by length). Figure 3 provides an overview of the FSAT. The leading edge of the model is installed $13 \mathrm{~cm}$ downstream of the inlet exit at a zero degree geometric angle of attack. The upper and lower boundary layers are tripped with zig-zag turbulator tape placed at $18 \%$ chord [2]. Throughout all testing the airfoil is bounded in the spanwise direction by sidewalls in order to ensure two-dimensional flow. The sidewalls are created from foam in order to minimize acoustic reflections on their surface. Further details regarding the FSAT are given by Pascioni et al [15]. 


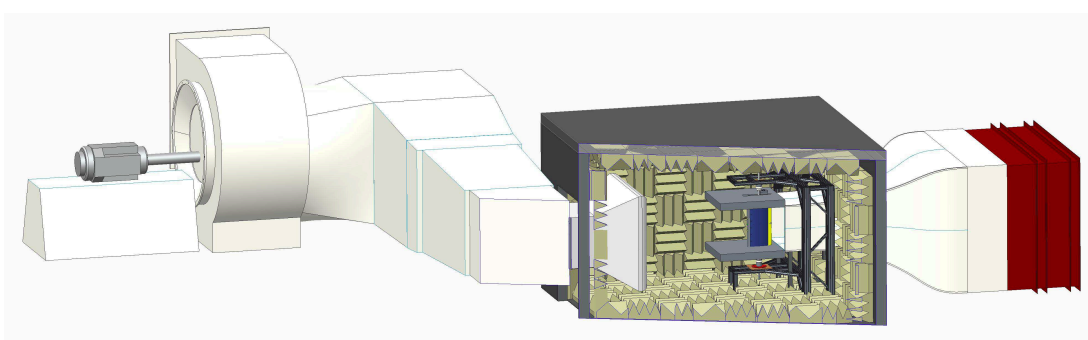

Figure 3: Overview of the FSAT facility. The foreground chamber wall is removed for illustration.

\subsection{Slot Characterization}

The slot jet speed is obtained from isentropic relations between plenum and freestream pressures such that

$$
U_{j}=\sqrt{2 R T_{0}\left(\frac{\gamma}{\gamma-1}\right)\left(1-\left(\frac{p_{\infty}}{p_{0}}\right)^{(\gamma-1) / \gamma}\right)}
$$

assuming zero (or very small) velocity within the plenum [2]. In Eq. (2.1), $R$ is the ideal gas constant and $\gamma$ is the ratio of specific heats, while the stagnation temperature, $T_{0}$, is measured by the RTD in the air delivery system, $p_{\infty}$ is measured by the Pitot-static probe in the free-stream, and $p_{0}$ is measured by pressure scanners via a pressure tap from the plenum. The jet velocity is held constant throughout testing to within $5 \%$ of the intended value. Using the jet velocity $U_{j}$, the jet mass flow rate $\dot{m}$ (measured via two Venturi meters), the freestream dynamic pressure $q_{\infty}$ and the model planform area $S$, the momentum coefficient is calculated as

$$
C_{\mu}=\frac{\dot{m} U_{j}}{q_{\infty} S}
$$

All pressure and temperature measurements used to determine jet velocity and momentum coefficients have $\pm 17 \mathrm{~Pa}$ and $\pm 0.1^{\circ} \mathrm{C}$ uncertainty, respectively.

\subsection{Acoustic Measurements}

The acoustic measurements taken throughout this study are based on both single microphone data and phased acoustic array methods. This section discusses single microphone and array beamforming techniques as well as the experimental setup, acquisition and processing parameters.

Single microphone auto-spectra can be obtained to view noise levels obtained over a frequency band of interest. However, since a single microphone simply provides the levels of all pressure incident on its diaphragm, it cannot distinguish between sources nor can it provide their locations. While considerably more complex, beamforming provides a method to obtain source locations and source field levels at the array center with background noise suppressed. Two methods of beamforming are considered in this document, delay-and-sum (DAS, or conventional) beamforming and the deconvolution approach for mapping acoustic sources (DAMAS). Details regarding their implementation can be found in [12] and [13], respectively.

\subsubsection{Phased Acoustic Array}

The phased array employed during testing consists of 55 microphones as shown in Figure 4. The array is designed for a frequency range of 1 to $16 \mathrm{kHz}$ with a constraint on maximum sidelobe level of $10 \mathrm{~dB}$ below the primary source. It is designed based on a reverse logarithmic spiral pattern as discussed in [16]. The array has 11 arms with 5 microphones on each arm. The innermost ring of microphones is located at a radial distance of $7.4 \mathrm{~cm}$ while the outermost ring is placed at $36.8 \mathrm{~cm}$ from the center.

The array frame is made of $1 \mathrm{~cm}$ thick 6061 aluminum in order to compromise strength (to ensure accurate placement of the microphones) and weight (to ensure that the array is easily mountable in a 


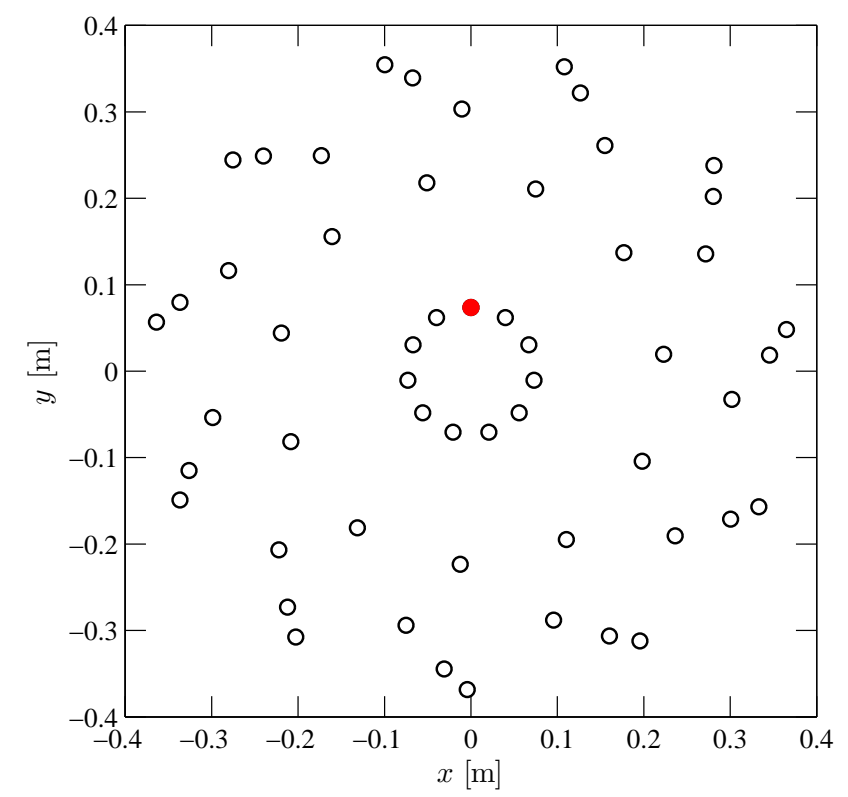

Figure 4: Array microphone locations. The array pattern is a reverse logarithmic spiral with center located at (0,0). Location marked by filled circle is used as the single microphone measurement location.

variety of ways). A skeletal pattern is cut into the aluminum by a water jet machine to reduce the effective area. The microphones are then placed onto ( 0.3 meter long) mounting rods to move them away from the frame and further reduce the effect of reflections. Finally, the frame is covered in $7.6 \mathrm{~cm}$ acoustic wedge foam to mitigate any remaining reflections.

\subsubsection{Experimental Setup}

Figure 5 shows a schematic of the tunnel acoustic setup. The phased acoustic array is installed on the same side as the CC slot jet at a distance of $1.22 \mathrm{~m}$ from the centerline of the model and vertically centered at the airfoil midspan. In the streamwise direction, the center of the array is aligned to the trailing edge of the model. The array contains 12 G.R.A.S. type 40BE 1/4-inch free-field microphones along with 43 Bruel and Kjær type 4958 1/4-inch microphones. All microphones are calibrated using a B\& K 4231 Sound Calibrator which operates at a frequency of $1 \mathrm{kHz}$.

\subsubsection{Acquisition and Processing Parameters}

Microphone data are acquired at $204,800 \mathrm{~Hz}$ for 30 seconds. Single microphone spectra are processed with $16 \mathrm{~Hz}$ binwidth employing a Hanning window with $75 \%$ overlap resulting in $3.2 \%$ autospectral random uncertainty. All spectral results in this document are scaled from a distance of 1.22 meters (distance from microphones to source) to a distance of 1 meter. A thin 2D shear layer correction [17] is applied to all data obtained with the wind tunnel turned on. This assumption may break down far downstream in the higher momentum coefficient cases due to the shear layer turning as a result of the high lift generated by the CC model.

\section{Results}

Acoustic measurements are made for five freestream Reynolds numbers from $R e_{c}=0$ to 1.3 million, and up to six momentum coefficients from $C_{\mu}=0$ to 0.10 (jet velocities up to $100 \mathrm{~m} / \mathrm{s}$, Mach 0.29). Higher 


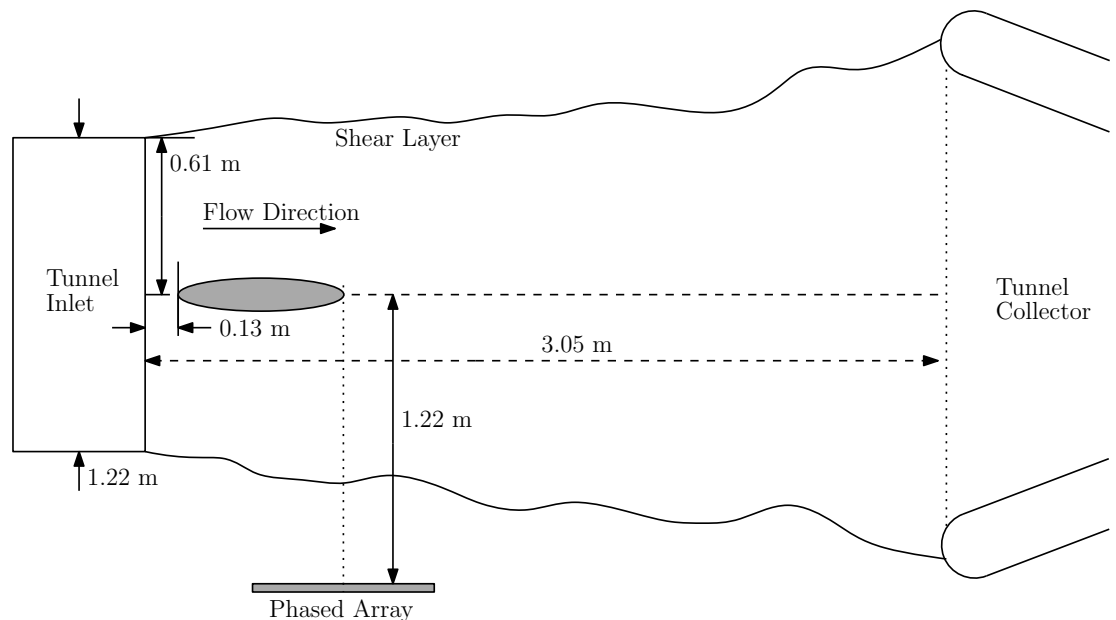

Figure 5: Florida State Aeroacoustic Tunnel with phased array and circulation control model installed. Note the the blowing slot is positioned on the same side as the array.

momentum coefficients are omitted at the high tunnel speeds to avoid over-pressurization of the internal plenum. This section begins by discussing single microphone spectra. It shows operating conditions where single microphone measurements are insufficient due to effective tunnel background noise. Then, it discusses phased acoustic array beamforming results attempting to remove the effects of the background noise.

\subsection{Single Microphone Results}

Single microphone spectra are a powerful tool in quickly determining noise levels over a wide range of frequencies. For the purposes of this section, a frequency range from $100 \mathrm{~Hz}$ to $80 \mathrm{kHz}$ is investigated. Note that the lower limit is below the anechoic chamber cut-on frequency $(250 \mathrm{~Hz})$ to show the shedding peak and the upper limit is set by the dynamic range of the microphone (location shown in Figure 4). Each spectral plot is shown in sound pressure level per Hertz $(\mathrm{SPL} / \mathrm{Hz})$ and contains all blowing conditions as well as a microphone noise floor. The plots also contain a "no model" noise floor which is taken with the wind tunnel running (and sidewalls installed) but no model in the tunnel, estimating the facility's effective background noise floor.

Initially, tunnel off $\left(R e_{c}=0\right)$ acoustics are investigated. For this case, jet Reynolds number $\left(R e_{J}\right.$, defined by slot height and jet velocity) is used instead of momentum coefficient due to the absence of a freestream. Results are shown in Figure 6. At the lowest jet Reynolds number, the noise generated from the model is below the noise floor of the microphone over a wide range of frequencies. For all jet Reynolds numbers, a peak occurs in the spectra from 500 to $900 \mathrm{~Hz}$. While this peak is at a constant frequency, its levels increase with jet velocity. When compared to hotwire tests performed pre-test, this appears to be a Helmholtz resonance of the plenum. Also apparent in the spectra is a high frequency hump which broadens, increases in frequency, and increases in magnitude as jet Reynolds number increases. One can deduce that this spectral behavior is due to slot-jet interaction noise since it is the only source present due to the absence of the freestream.

Results from tests at several different chord Reynolds numbers and blowing conditions are considered next. These data are shown in Figures 7 - 10. For all cases at the no blowing condition $\left(C_{\mu}=0\right)$ the effective background noise is significant for all frequencies. It should be noted, that for all the no blowing cases, a peak in the spectra exists (below the tunnel's lowest anechoic frequency, an example of which is shown in Figure 10) which corresponds to bluff body shedding. For the lowest chord Reynolds number $\left(R e_{c}=3.2 e 5\right.$, Figure 7) the spectral levels for all blowing conditions are (at best) marginally above the tunnel and microphone noise floors. At the highest chord Reynolds number $\left(R e_{c}=1.3 e 6\right.$, Figure 10) a similar trend is noted (especially at low frequencies) due to the inability to achieve significantly large momentum coefficients at this tunnel speed. 


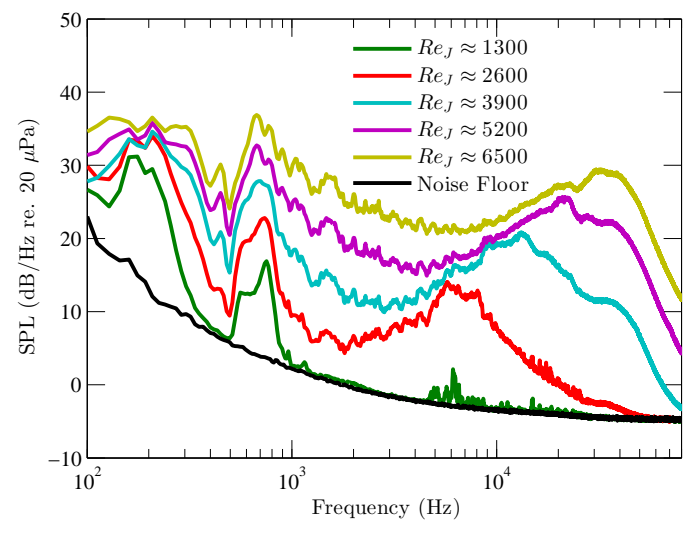

Figure 6: Spectral data from a single microphone above the trailing edge on the same side as the blowing slot at $R e_{c}=0$.

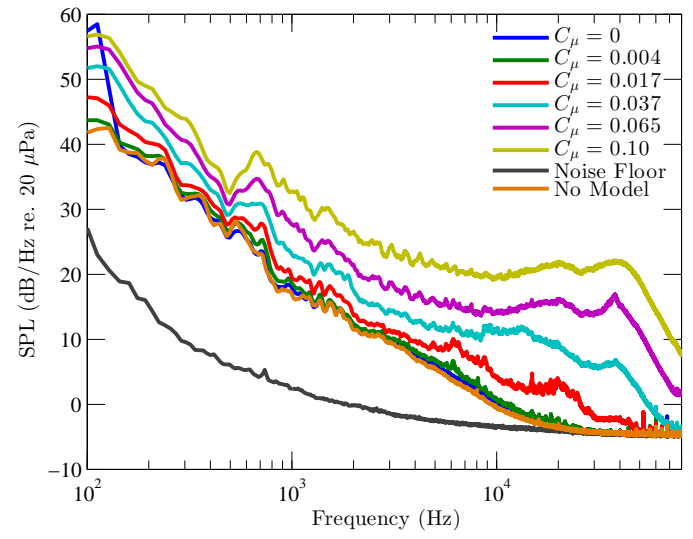

Figure 8: Spectral data from a single microphone above the trailing edge on the same side as the blowing slot at $R e_{c}=6.5 e 5$.

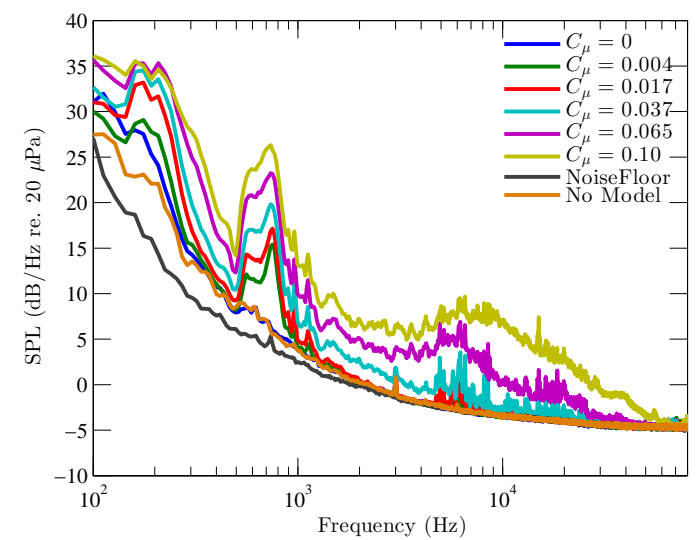

Figure 7: Spectral data from a single microphone above the trailing edge on the same side as the blowing slot at $R e_{c}=3.2 e 5$.

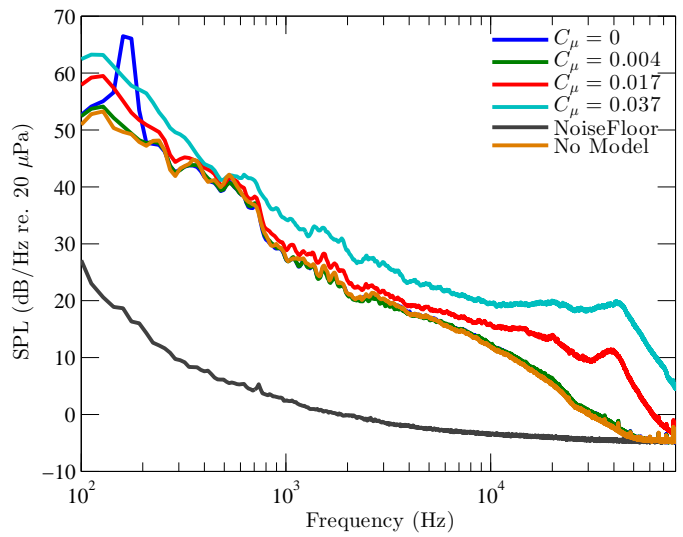

Figure 9: Spectral data from a single microphone above the trailing edge on the same side as the blowing slot at $R e_{c}=9.7 e 5$.

(19)

At the lowest blowing conditions for the other two chord Reynolds numbers $\left(R e_{c}=6.5 e 5\right.$ and $R e_{c}=$ $9.7 e 5$, Figures 8 and 9), the effective tunnel background noise remains a significant source of acoustics. However, unlike the previous cases, the higher momentum coefficient cases show significant noise being generated by the model. It is shown in Figures 8 and 9 that from middle to high frequencies, the noise increases with increasing coefficient of momentum. This suggests increased noise generated by the slot-jet interacting with the slot lip as well as higher passive slot noise [6]. However, additional noise sources may be present.

Due to the significant levels of effective background noise shown in the single microphone spectra, another method will be investigated to obtain accurate model-generated-noise spectra. Two- and three-microphone methods were considered; however, the background noise is generated by the tunnel and may be correlated between microphone pairs. These methods remove only uncorrelated self noise. Therefore, phased acoustic array beamforming is used for this purpose.

\subsection{Beamforming}

Unlike single microphone measurements, a phased array allows for the identification of individual sources by digitally focusing all the microphones to a single point. By using the ability to focus on certain locations 


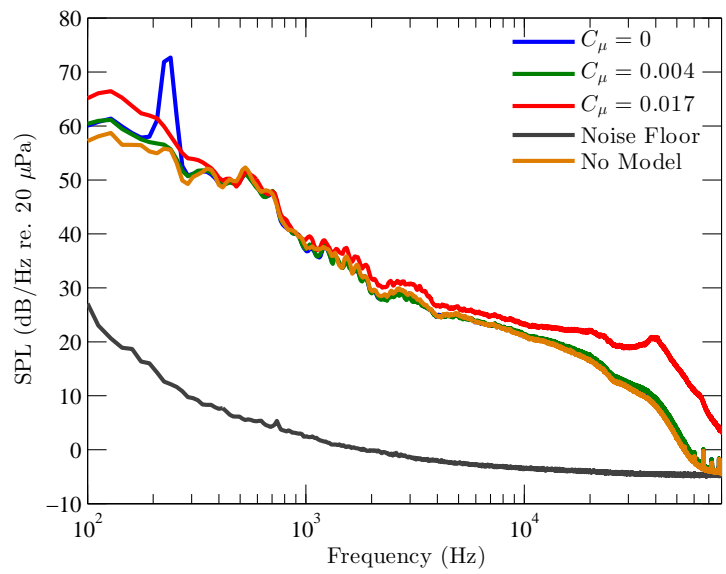

Figure 10: Spectral data from a single microphone above the trailing edge on the same side as the blowing slot at $R e_{c}=1.3 e 6$.
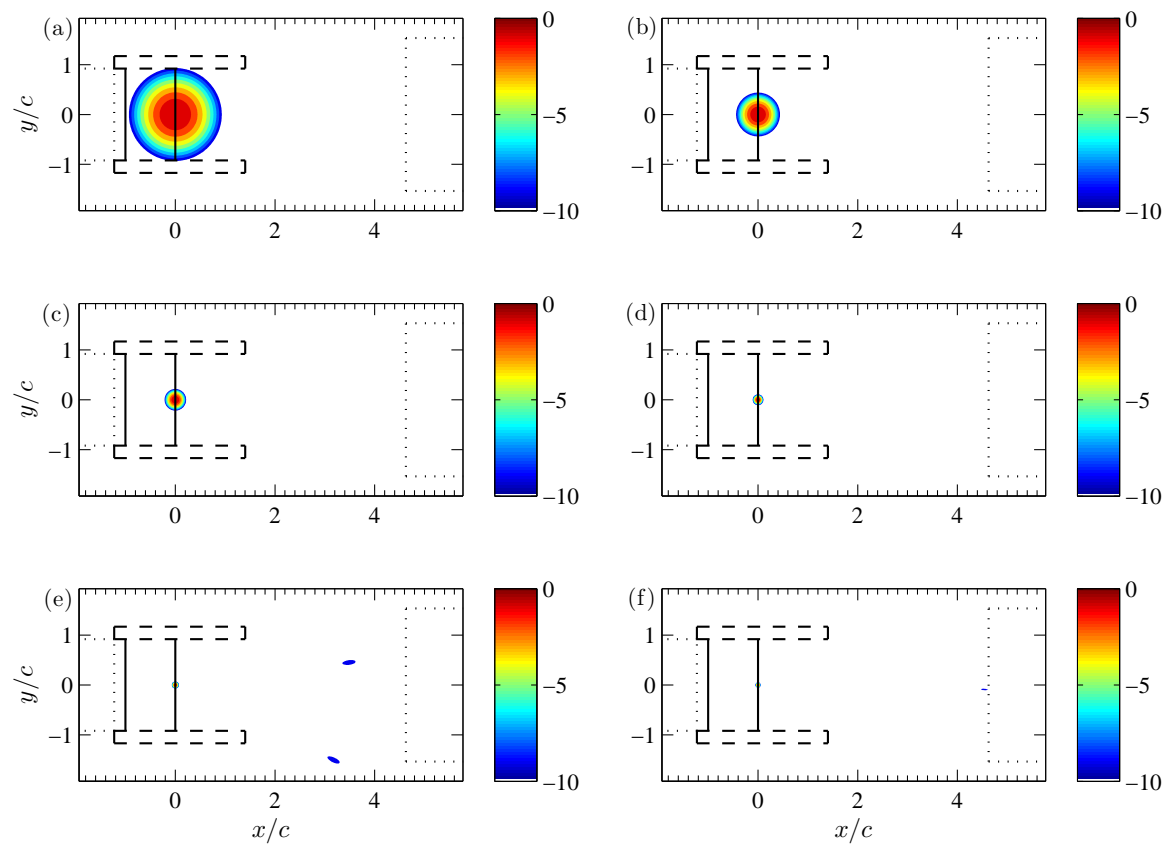

Figure 11: Theoretical point spread functions for the array pattern used during this study. (a) $1 \mathrm{kHz}$, (b) $2 \mathrm{kHz},(\mathrm{c}) 4 \mathrm{kHz}$, (d) $8 \mathrm{kHz}$, (e) $12 \mathrm{kHz}$, and (f) $16 \mathrm{kHz}$.

and summing up only the relevant components, spectral levels can be obtained which suppress effective background noise. Unfortunately, array design constraints and microphone bandwidth limit the frequency range over which beamforming is possible from approximately 1 to $16 \mathrm{kHz}$. Fortunately, this frequency range overlaps a large portion of the relevant single microphone data.

Due to its relative simplicity (and small computation time), delay-and-sum beamforming (DAS) is investigated initially. The array point spread functions (PSF) for the frequencies discussed are shown in Figure 11, with the source located at the center span of the trailing edge. In this and all other beammaps in this document, the dotted lines on the left indicate the location of the inlet and on the right indicate the collector, 
the dashed lines on top and bottom indicate the sidewall locations and the solid vertical lines define the leading (left) and trailing (right) edges of the model, as shown in Figure 12. Flow goes from left to right in these beammaps.

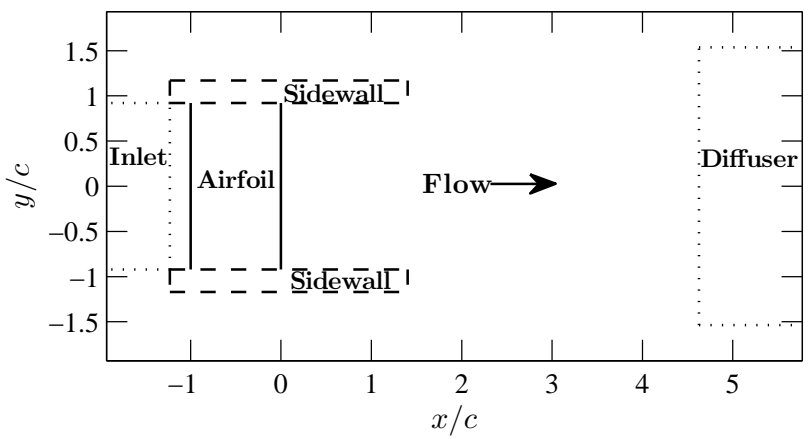

Figure 12: Schematic labeling relevant objects in beammaps.

\subsubsection{Delay-and-Sum}

Initially, three cases are investigated which provide a representative sample of the complexities associated with circulation control. These cases are 1.) zero freestream velocity (zero chord Reynolds number, $R e_{c}=0$ ), $100 \mathrm{~m} / \mathrm{s}$ jet velocity (6500 jet Reynolds number, $\left.R e_{J}=6500\right), 2$.) $R e_{c}=6.5 e 5$, zero jet velocity (zero coefficient of momentum, $C_{\mu}=0$ ), 3.) $R e_{c}=6.5 e 5, C_{\mu}=0.10$ (jet velocity approximately 5 times freestream velocity). By studying the beammaps from these cases, insight into the sources, locations, and conditions for different external noise sources is observed. These data will then be used to determine accurate integration regions to acquire overall CC noise with external sources removed.

Figure 13 shows the DAS beammaps for frequencies of $1,2,4,8,12$, and $16 \mathrm{kHz}$ for the $R e_{c}=0$, maximum blowing $\left(R e_{J}=6500\right)$ case. At the lowest frequency $(1 \mathrm{kHz})$, the primary source of noise is centered around the model trailing edge, which could indicate curvature noise [6]. At the middle frequencies of 2 and $4 \mathrm{kHz}$, the primary noise source is at the slot-jet exit (slightly upstream of the trailing edge); indicative of slot-jet interaction or passive slot noise [6]. As the frequency increases, the sources move toward the sidewalls. This suggests jet/sidewall interaction becoming more influential as frequency increases.

Figure 14 shows DAS beammaps for the $R e_{c}=6.5 e 5, C_{\mu}=0$ case at the same frequencies. Here, the effective background noise is clearly significant at all frequencies. At the lowest frequency, the collector is the dominant noise source with a source of unknown origin occurring at the model's leading edge. At low frequencies $(2$, and $4 \mathrm{kHz})$ the noise generated from the collector continues to be the dominant noise source. As frequency increases ( $4 \mathrm{kHz}$ and higher) the sidewall noise near the leading edge of the model becomes the dominant source. These beammaps confirm the result shown by the single microphone spectra discussed previously; the effective background noise appears to be the significant noise source over all relevant frequencies.

Finally, Figure 15 investigates the $R e_{c}=6.5 e 5, C_{\mu}=0.10$ case. The dominant noise source for all frequencies is the jet/sidewall interaction.

The three general sources of background and installation noise are revealed in these experiments: 1.) collector noise, 2.) sidewall noise (near the leading edge), and 3.) jet/sidewall interaction noise. To isolate the CC noise (not related to installation or tunnel effects), a region which removes all of these external noise sources should be employed. In the streamwise direction one needs to include only the airfoil itself and regions slightly downstream (to include jet noise). By limiting the region in this manner the collector noise is removed. In the spanwise direction, the sidewall interaction noise must be excluded. At a frequency of 2 $\mathrm{kHz}$, only the middle $0.44 \mathrm{~m}$ of the airfoil span is included. The integration region chosen based on these criteria (assuming an origin at center span on the model's trailing edge) is -1.2 to 0.4 chords (-0.6 to 0.2 $\mathrm{m})$ in the streamwise direction and -0.43 to 0.43 chords $(-0.22$ to $0.22 \mathrm{~m})$ in the spanwise direction. These 

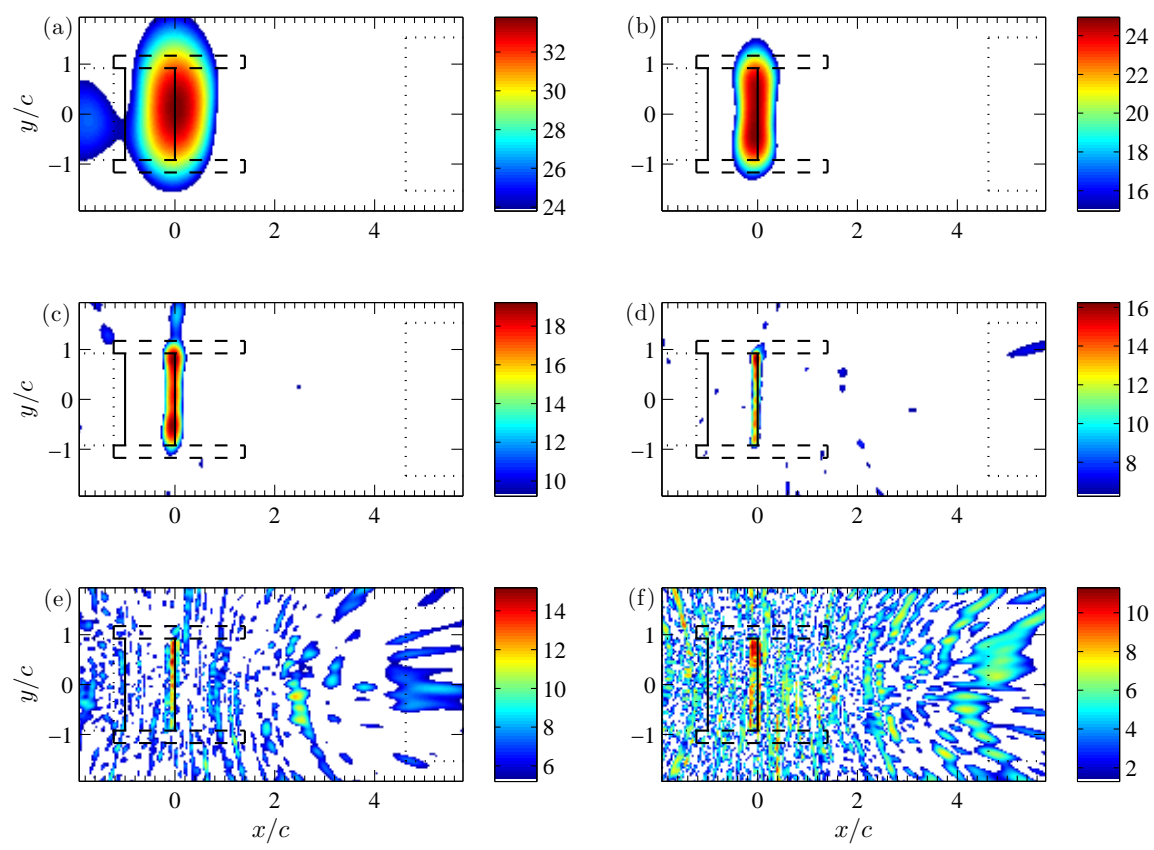

Figure 13: Beammaps for the $R e_{c}=0, R e_{J}=6500$ case. (a) $1 \mathrm{kHz}$, (b) $2 \mathrm{kHz}$, (c) $4 \mathrm{kHz}$, (d) $8 \mathrm{kHz},(\mathrm{e}) 12 \mathrm{kHz}$, and (f) 16 $\mathrm{kHz}$.
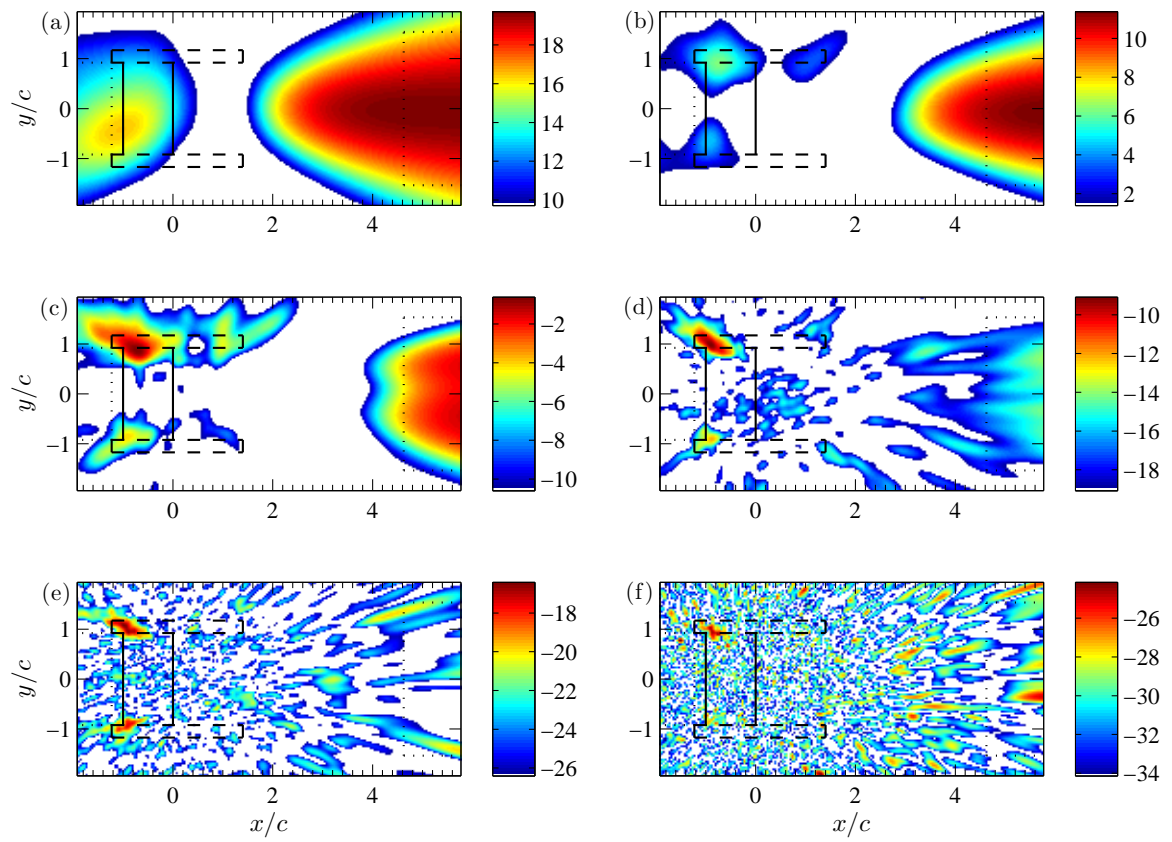

Figure 14: Beammaps for the $R e_{c}=6.5 e 5, C_{\mu}=0$, no blowing, case. (a) $1 \mathrm{kHz}$, (b) $2 \mathrm{kHz}$, (c) $4 \mathrm{kHz},(\mathrm{d}) 8 \mathrm{kHz},(\mathrm{e}) 12 \mathrm{kHz}$, and (f) $16 \mathrm{kHz}$. 

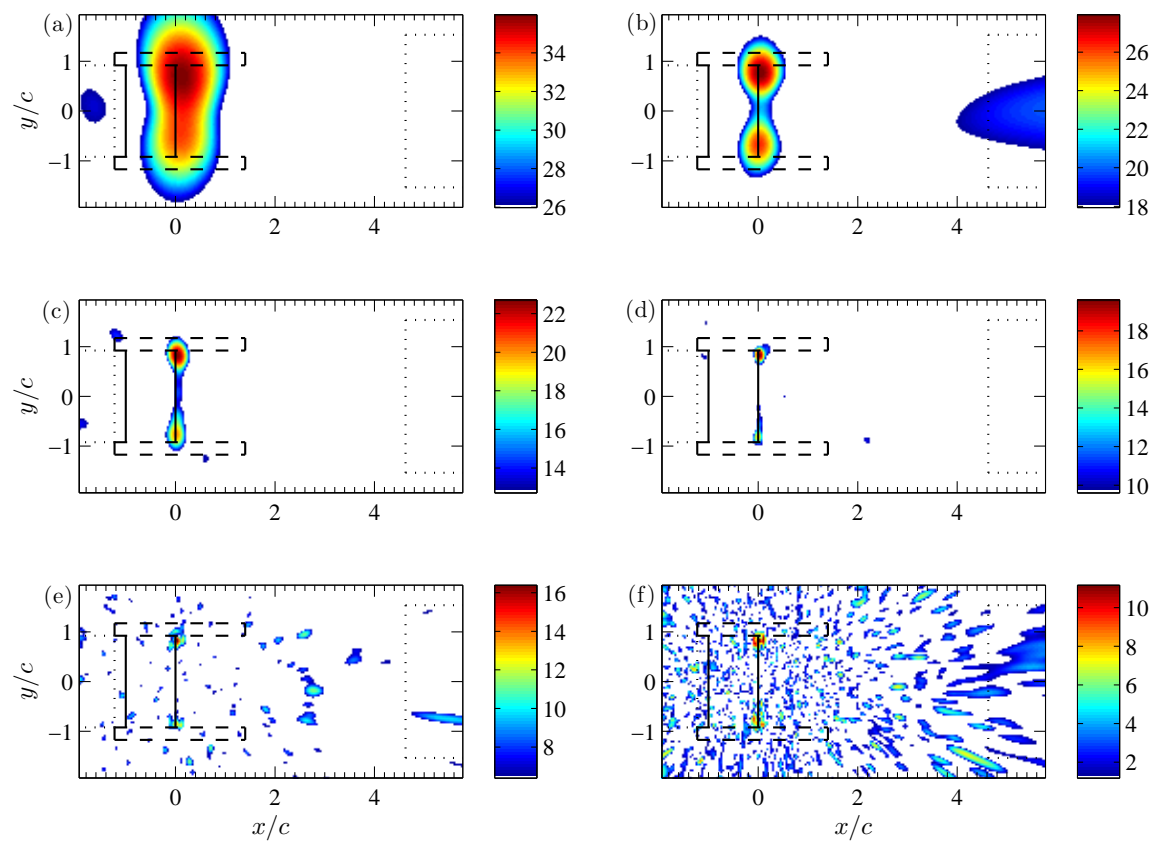

Figure 15: Beammaps for the $R e_{c}=6.5 e 5, C_{\mu}=0.10$ case. (a) $1 \mathrm{kHz}$, (b) $2 \mathrm{kHz}$, (c) $4 \mathrm{kHz}$, (d) $8 \mathrm{kHz}$, (e) $12 \mathrm{kHz}$, and (f) 16 $\mathrm{kHz}$.

results are then scaled to a unit meter span (from 0.44 meters) and a unit meter distance (at $90^{\circ}$ from the chordwise and spanwise directions) in order to compare to the single microphone results. Integrated spectra for all DAS results are provided with shaded uncertainty bounds.

Uncertainty in the integrated DAS spectra is also estimated via 1000 iteration Monte-Carlo simulations [18]. For these simulations the DAS beamformer is perturbed about: 1.) microphone position $(x, y$, and $z$ coordinates by $1 \mathrm{~cm}), 2$.) scanning plane height $(2 \%), 3$.$) microphone sensitivity (10 \%), 4$.$) temperature$ (1\%), and 5.) cross-spectral uncertainty. Note that the simulations were not perturbed about shear layer parameters.

Due to computational expense and the distinct lack of peaks, spectra for the DAS (which are expensive due to the uncertainty estimate) and DAMAS (which is expensive due to calculation of the shear layer correction for each resolution) are gathered at the 1/12th octave band centers instead of the $16 \mathrm{~Hz}$ resolution used in the single microphone spectra. Note that the cross-spectral matrices are generated with $16 \mathrm{~Hz}$ bins; instead of beamforming at all narrow-band frequencies we compute the results only at the $1 / 12$ th octave band center frequencies and no integration is performed. The $R e_{c}=3.2 e 5$ cases are not included for the beamforming cases due to non-physical behavior caused by their proximity to the microphone noise floor.

The tunnel off $\left(R e_{c}=0\right)$ integrated spectra are compared to the single microphone result in Figure 16. Overall, the DAS results are several decibels lower than the single microphone due to the exclusion of extraneous sources. The shape of the spectra over this range of frequencies are quite similar for both data types, confirming that the conclusions drawn from the single microphone data for this case are indeed from the $\mathrm{CC}$ and not background noise.

Turning attention to those cases with considerably more effective background noise the $R e_{c}=6.5 e 5$, $R e_{c}=9.7 e 5$, and $R e_{c}=1.3 e 6$ single microphone and DAS cases are compared in Figures 17, 18, and 19, respectively. In the $R e_{c}=6.5 e 5$ cases a significant reduction in overall levels from the single microphone results are shown. At high frequencies the no blowing and lowest blowing $\left(C_{\mu}=0\right.$ and $C_{\mu}=0.004$, respectively) cases are still saturated by the effective wind tunnel background noise and show large uncertainty 


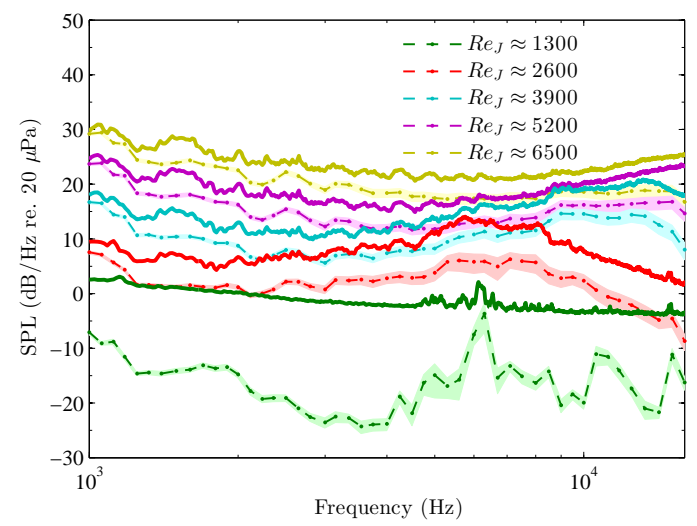

Figure 16: Spectral data from a single microphone $(16 \mathrm{~Hz}$ binwidth, solid line with no markers) compared to DAS integrated spectra (1/12th octave bands centers, dashed lines with dot markers) with shading showing DAS 95\% uncertainty based on 1000 iteration Monte Carlo simulations at $R e_{c}=0$.

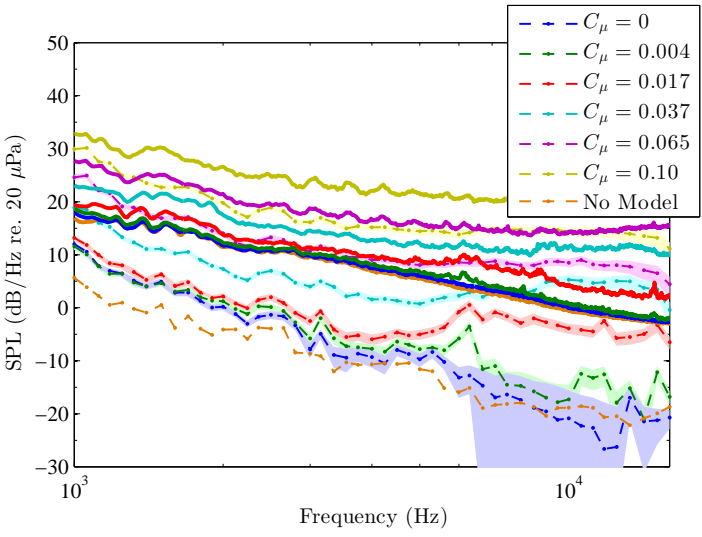

Figure 17: Spectral data from a single microphone $(16 \mathrm{~Hz}$ binwidth, solid line with no markers) compared to DAS integrated spectra (1/12th octave band centers, dashed lines with dot markers) with shading showing DAS 95\% uncertainty based on 1000 iteration Monte Carlo simulations at $R e_{c}=6.5 e 5$.

(in some areas even larger than the mean value). It is also shown that at low frequencies for the lower blowing conditions $\left(C_{\mu}=0, C_{\mu}=0.004\right.$, and $\left.C_{\mu}=0.017\right)$ the levels are quite similar. By using DAS, the higher blowing cases $\left(C_{\mu}=0.037, C_{\mu}=0.065\right.$, and $\left.C_{\mu}=0.10\right)$ are much more significantly differentiated from the effective background noise. It should also be noted (similar to the $R e_{c}=0$ case) that the shape of the spectra vary little from the single microphone to the DAS results.

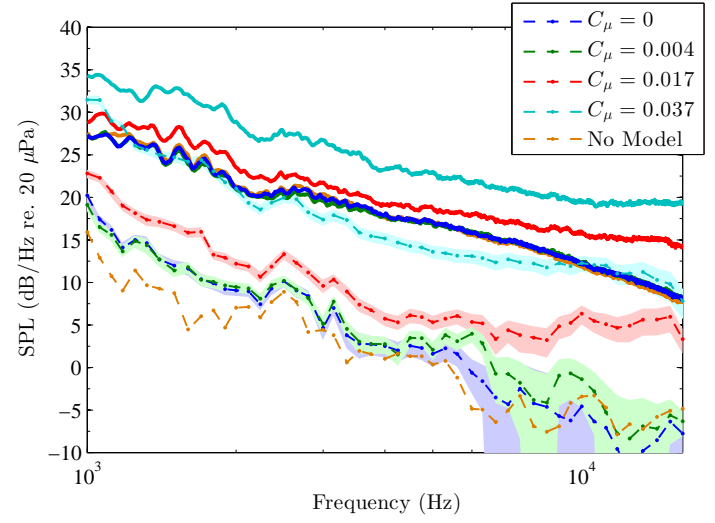

Figure 18: Spectral data from a single microphone $(16 \mathrm{~Hz}$ binwidth, solid line with no markers) compared to DAS integrated spectra (1/12th octave band centers, dashed lines with dot markers) with shading showing DAS $95 \%$ uncertainty based on 1000 iteration Monte Carlo simulations at $R e_{c}=9.7 e 5$.

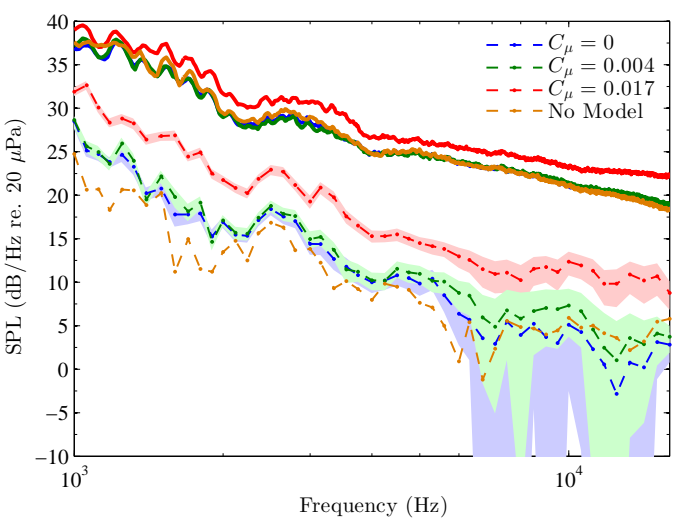

Figure 19: Spectral data from a single microphone $(16 \mathrm{~Hz}$ binwidth, solid line with no markers) compared to DAS integrated spectra $(1 / 12$ th octave band centers, dashed lines with dot markers) with shading showing DAS $95 \%$ uncertainty based on 1000 iteration Monte Carlo simulations at $\operatorname{Re}_{c}=1.3 e 6$.

In the $R e_{c}=9.7 e 5$ and $R e_{c}=1.3 e 6$ cases (Figures 18 and 19) the changes from single microphone to DAS results are quite similar to those shown in the $R e_{c}=6.5 e 5$ case. Once again, a significant decrease in overall levels for the DAS results is shown. For the no and lowest blowing conditions $\left(C_{\mu}=0\right.$, and $\left.C_{\mu}=0.004\right)$ the uncertainty is once again larger than the mean value at high frequencies and at low frequencies they converge due to the (unremovable) reflected noise. Unlike the $R e_{c}=6.5 e 5$ case, the DAS removes enough effective background noise from the $C_{\mu}=0.017$ case that it is significantly above the tunnel noise floor. 
For the higher blowing condition $\left(C_{\mu}=0.037\right)$ in the $R e_{c}=9.7 e 5$ case it is again observed that, while the absolute levels are reduced, the shape of the spectra is unchanged.

\subsubsection{Deconvolution Approach Mapping Acoustic Sources}

DAMAS removes the influence of the point spread function from the DAS beamforming result. By doing so it can provide accurate source locations and levels. However, because DAMAS relies on solving an inverse problem [13] care must be taken when choosing scanning region bounds, number of iterations, and grid resolution. Ideally, one would simply maximize each of these items. Unfortunately, this is not possible due to the computational expense associated with DAMAS.

Each of these parameters is studied from simulated acoustic fields and experimental results prior to processing. The grid resolution and scanning region bounds must be balanced with computational time to obtain integrated spectra in a reasonable time-frame. It is observed (in pre-processing) that a field spanning -2 to 6 chords $(-1.0$ to $3.1 \mathrm{~m})$ in the streamwise direction and -1.7 to 1.7 chords $(-0.9$ to $0.9 \mathrm{~m})$ in the spanwise direction is reasonable with a grid resolution of $20 \%$ of the $3 \mathrm{~dB}$ beamwidth for frequencies below $6 \mathrm{kHz}$. Above $6 \mathrm{kHz}$, the region size and resolution (relative to the beamwidth) are reduced due to the decreasing beamwidth. For frequencies above $6 \mathrm{kHz}, 30 \%$ of the beamwidth is used along with a scanning region from -1.5 to 1.5 chords $(-0.75$ to $0.75 \mathrm{~m})$ in both the streamwise and spanwise directions. The scanning regions are chosen to be larger than the integration region because sources outside of the DAMAS solution are erroneously located on the edges of the region. Figure 20 shows the scanning region bounds for both instances. For the experimental cases studied, the results converge to a solution by 1000 iterations. While the scanning region is varied over the range of frequencies, the integration region for the spectra remains the same as in the DAS beamforming. Spectral results are, once again, scaled from 0.44 meter span to a unit meter span and from a 1.22 meter distance for a unit meter distance for comparison purposes.

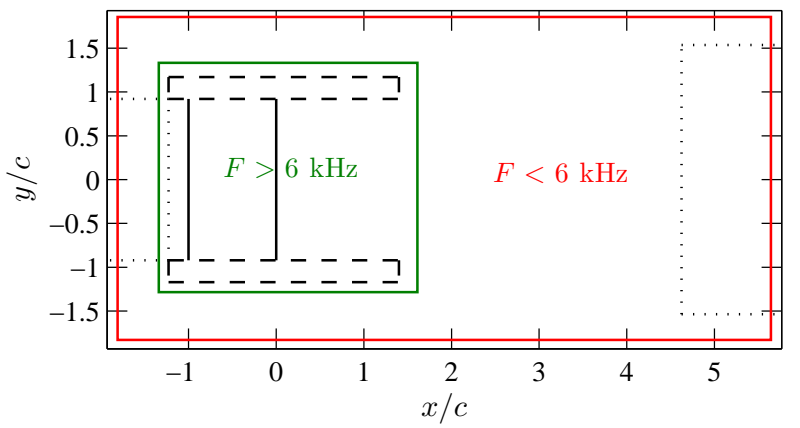

Figure 20: Schematic showing the scanning region bounds used in DAMAS for all frequency ranges.

Investigating the three cases discussed previously provides similar source locations. Figure 21 shows the $R e_{c}=0, R e_{J}=6500$ case using DAMAS. As shown in the DAS result, the primary source at $1 \mathrm{kHz}$ occurs at the trailing edge of the model, suggesting curvature noise [6]. The movement of the source upstream to the slot-jet exit at the middle frequencies is shown more clearly in DAMAS than in DAS. Contrary to the DAS result, the high frequency noise does not appear to be located heavily near the sidewalls and, instead, is distributed in a manner similar to the middle frequencies. This suggests that slot-jet interaction and passive slot noise are the dominant source for all but the lowest frequency [6].

Figure 22 shows DAMAS applied to the $R e_{c}=6.5 e 5, C_{\mu}=0$ case. At $1 \mathrm{kHz}$ the DAMAS result shows the same properties as the DAS; the primary noise source occurs at the collector with reflected noise near the leading edge of the airfoil. As frequency increases, the DAMAS result shows much more clearly that the primary source of noise is the sidewall near the model. While the DAS results at the highest frequencies show noise sources scattered throughout the domain, the DAMAS results remove these sidelobes and identify the dominant noise source as the sidewalls.

As shown in Figure 23 at the highest blowing condition, $C_{\mu}=0.10$, the DAMAS result shows the same trends as the DAS, again with significantly reduced sidelobe noise at high frequencies. Unlike the $R e_{c}=0$ 

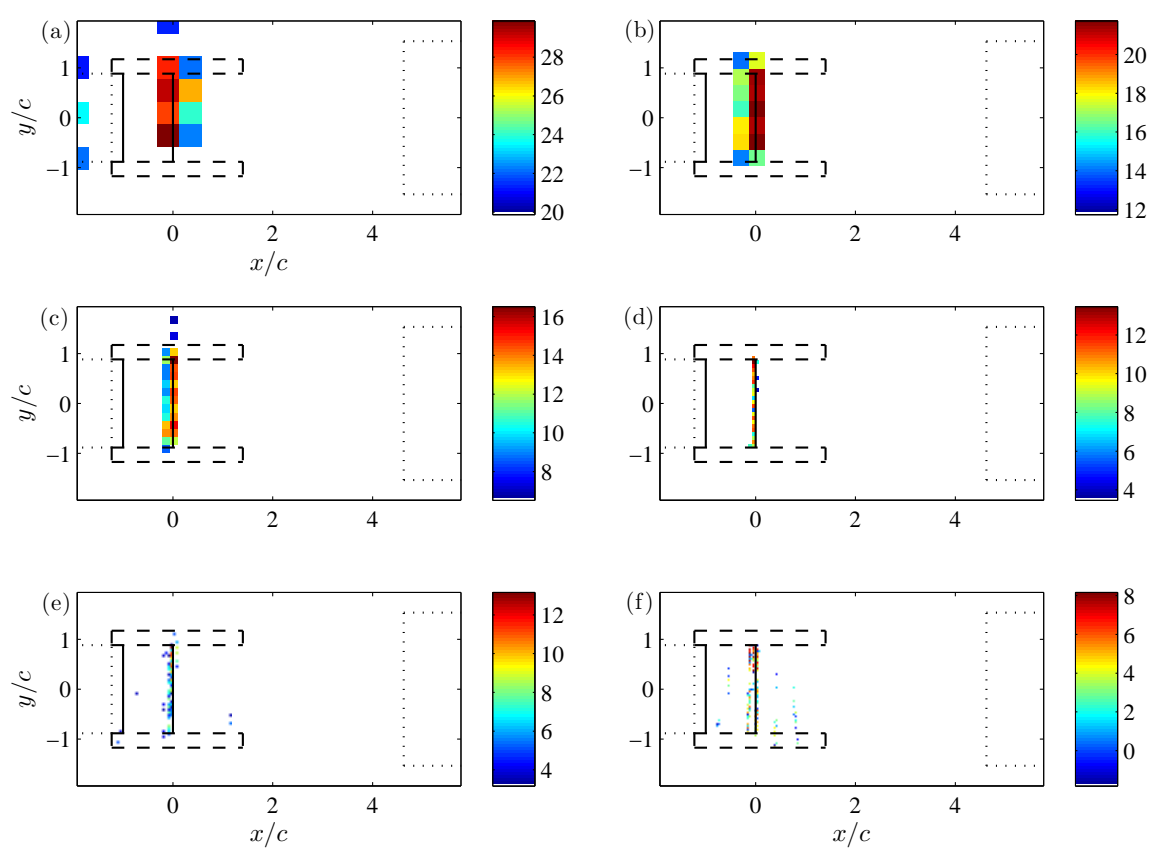

Figure 21: DAMAS beammaps for the $R e_{c}=0, R e_{J}=6500$ case. (a) $1 \mathrm{kHz}$, (b) $2 \mathrm{kHz}$, (c) $4 \mathrm{kHz}$, (d) $8 \mathrm{kHz},(\mathrm{e}) 12 \mathrm{kHz}$, and (f) $16 \mathrm{kHz}$.
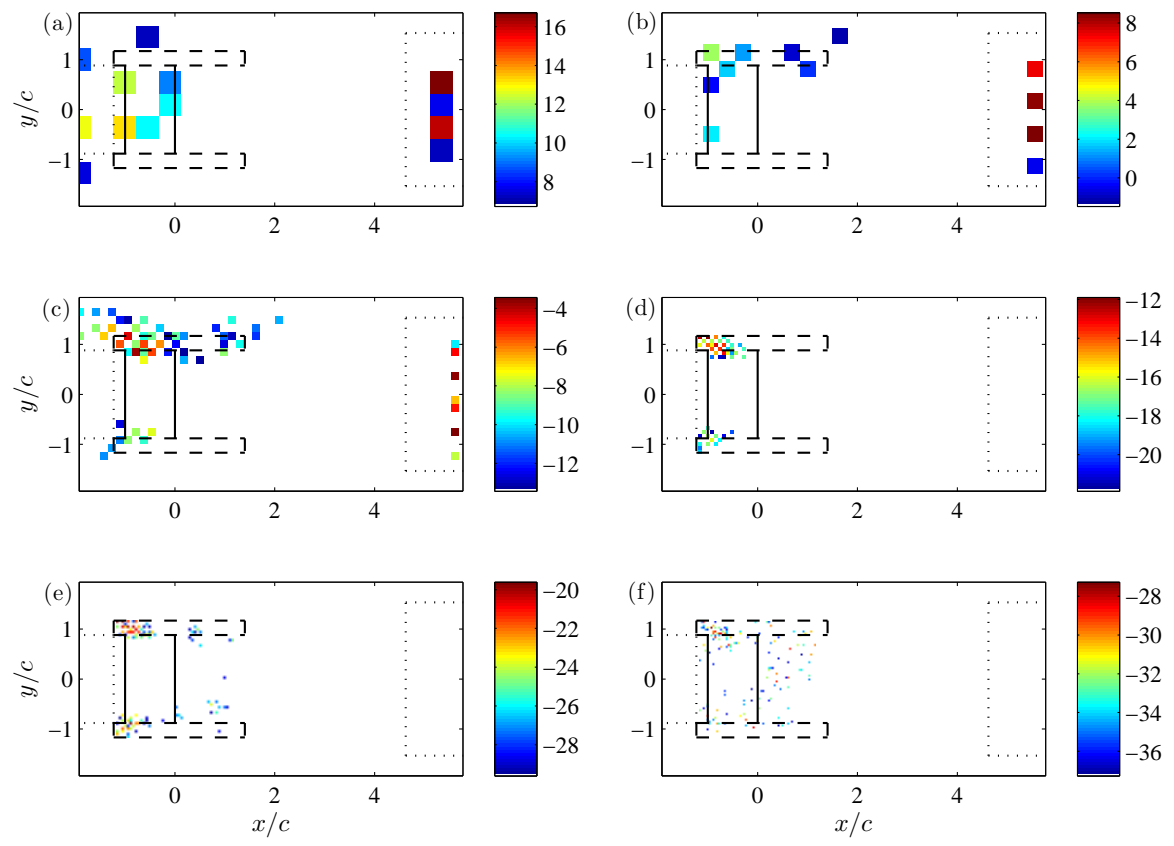

Figure 22: DAMAS beammaps for the $R e_{c}=6.5 e 5, C_{\mu}=0$ case. (a) $1 \mathrm{kHz}$, (b) $2 \mathrm{kHz}$, (c) $4 \mathrm{kHz}$, (d) $8 \mathrm{kHz}$, (e) $12 \mathrm{kHz}$, and (f) $16 \mathrm{kHz}$. 

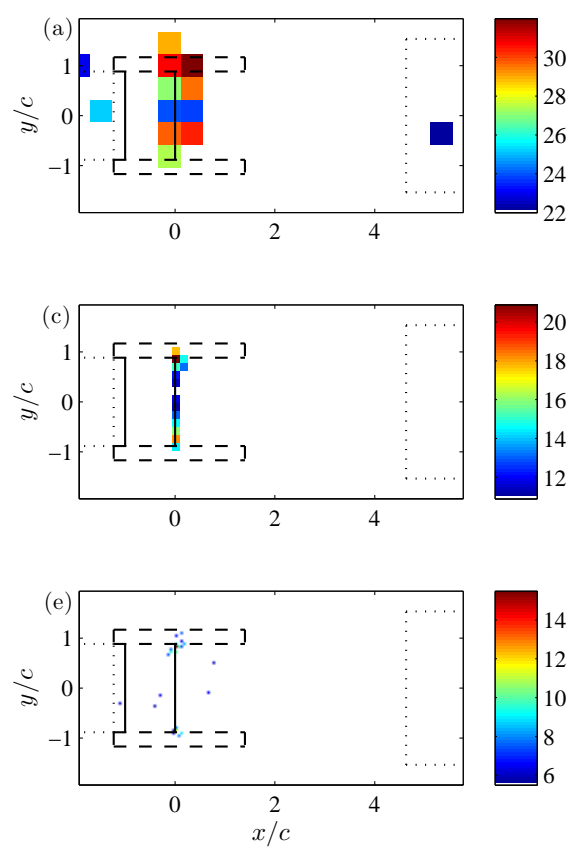
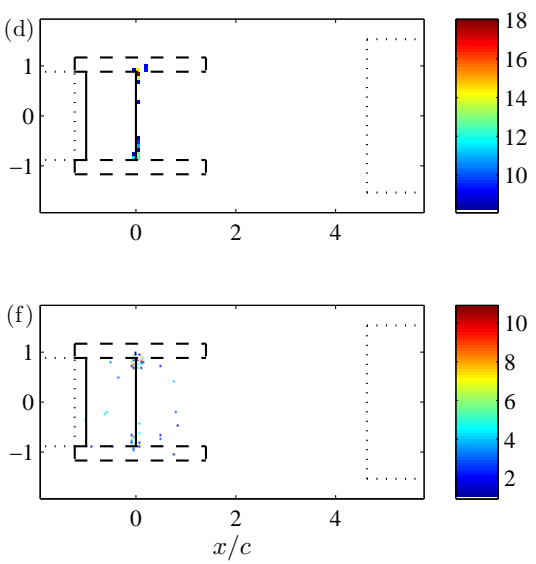

Figure 23: DAMAS beammaps for the $R e_{c}=6.5 e 5, C_{\mu}=0.10$ case. (a) $1 \mathrm{kHz}$, (b) $2 \mathrm{kHz},(\mathrm{c}) 4 \mathrm{kHz},(\mathrm{d}) 8 \mathrm{kHz},(\mathrm{e}) 12 \mathrm{kHz}$, and (f) $16 \mathrm{kHz}$.

case, the sources at 2 and $4 \mathrm{kHz}$ do not move as far upstream. This difference can be attributed to the changing dominant noise source mechanism from slot-jet [6] in the case without a free-stream to jet-sidewall interaction in the $R e_{c}=6.5 e 5$ case.

Comparing the DAMAS results to the DAS results suggests that DAMAS spectral results are likely to be more accurate than DAS, especially at higher frequencies, due to its exclusion of sidelobes. Figures $24,25,26$, and 27 provide spectral comparisons between the DAMAS and DAS beamformer results for all free-stream Reynolds numbers and momentum coefficients.

In the $R e_{c}=0$ cases (Figure 24) the DAS result shows slightly higher levels than DAMAS for moderate to high frequencies. The difference between DAS and DAMAS over these frequencies decreases with increasing momentum coefficient. This behavior is likely due to the tendency for the sidelobes to have a greater effect when the overall levels are lower.

With the flow at prescribed Reynolds numbers (Figures 25, 26, and 27) several similarities to the DAS results are shown. The zero and lowest $\left(C_{\mu}=0.004\right)$ momentum coefficients remain contaminated by effective background noise. As with the DAS results, the DAMAS output removes enough external noise from the $C_{\mu}=0.017, R e_{c}=9.7 e 5$ and $R e_{c}=1.3 e 6$ cases to distinguish them from the background (no model) noise (compared to the single microphone data). Also, like DAS, the effective background noise continues to be significant at low to middle frequencies in the $C_{\mu}=0.017, R e_{c}=6.5 e 5$ case. The trend noted in the $R e_{c}=0$ cases continues with the DAMAS results being slightly lower than the DAS at high frequencies.

Even using DAMAS the spectral results show that the two lowest blowing conditions $\left(C_{\mu}=0\right.$ and $\left.C_{\mu}=0.004\right)$ are not appreciably above the no model case for any of the free-stream Reynolds numbers. A scaling analysis is now performed on the suitable acoustic data.

\subsection{Acoustic Comparison to NACA 0012}

With all non-CC noise sources suppressed, the overall sound pressure levels (OASPL) from the CC experiments are compared to those of a NACA 0012 airfoil. NAFNoise [19] is employed to match the 


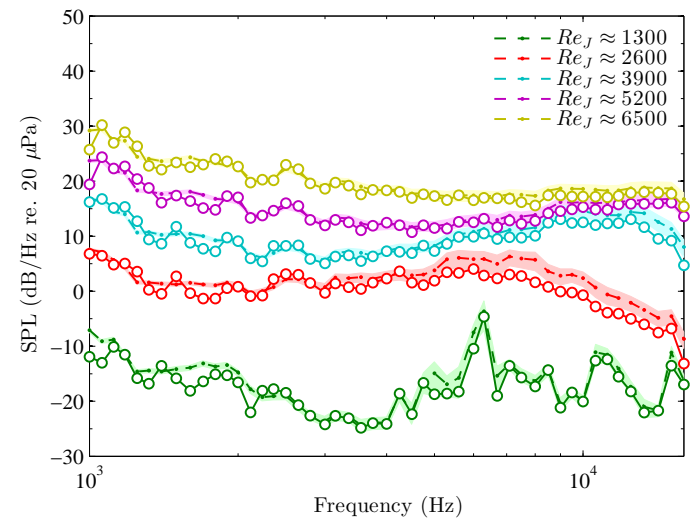

Figure 24: Spectral data from DAS (dashed lines with dot markers) and DAMAS (solid line with hollow markers) integrated spectra at the $1 / 12$ th octave bands centers with shading showing DAS 95\% uncertainty based on 1000 iteration Monte Carlo simulations at $R e_{c}=0$.

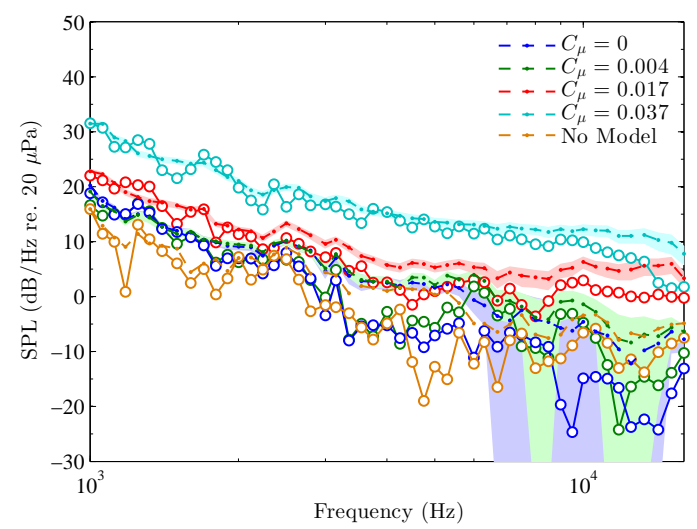

Figure 26: Spectral data from DAS (dashed lines with dot markers) and DAMAS (solid line with hollow markers) integrated spectra at the 1/12th octave bands centers with shading showing DAS $95 \%$ uncertainty based on 1000 iteration Monte Carlo simulations at $R e_{c}=9.7 e 5$.

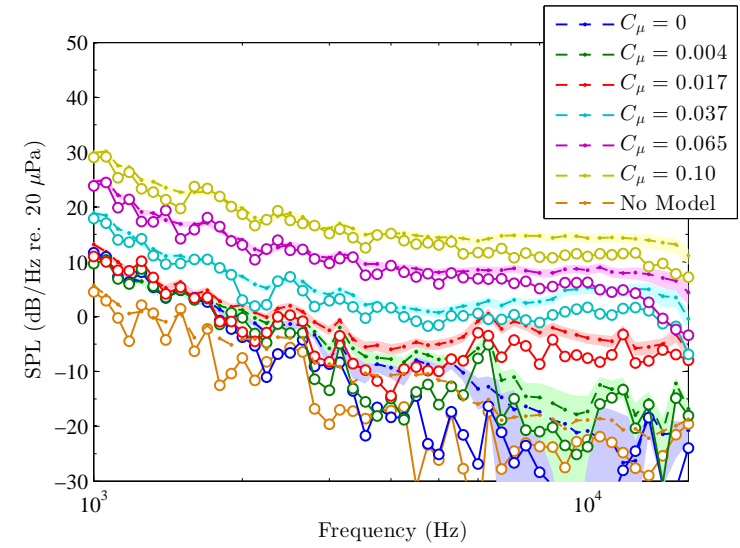

Figure 25: Spectral data from DAS (dashed lines with dot markers) and DAMAS (solid line with hollow markers) integrated spectra at the $1 / 12$ th octave bands centers with shading showing DAS $95 \%$ uncertainty based on 1000 iteration Monte Carlo simulations at $R e_{c}=6.5 e 5$.

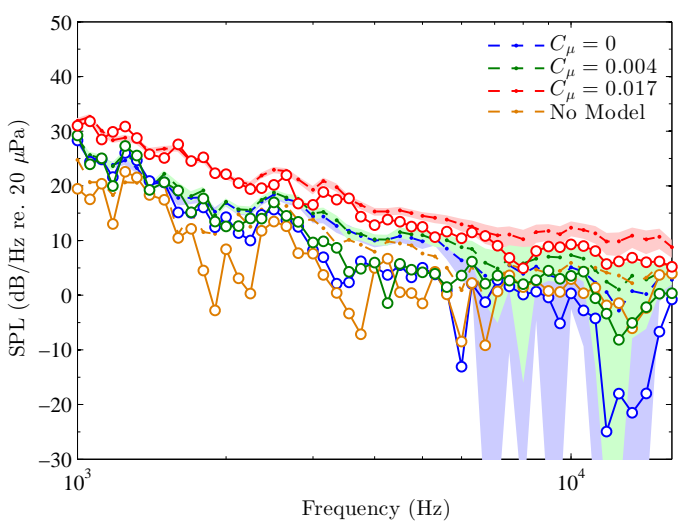

Figure 27: Spectral data from DAS (dashed lines with dot markers) and DAMAS (solid line with hollow markers) integrated spectra at the $1 / 12$ th octave bands centers with shading showing DAS $95 \%$ uncertainty based on 1000 iteration Monte Carlo simulations at $R e_{c}=1.3 e 6$.

specific parameters of the CC airfoil experiment, namely, airfoil geometric parameters (chord, span, observer distance, and observer location), and free-stream flow parameters (density, velocity, Mach number, Reynolds number, and lift coefficient). Lift coefficient is matched by varying the angle of attack in the NACA 0012 estimate. In the estimate, boundary layer trips are placed at $0.2 x / c$ on the upper and lower surfaces, corresponding to the locations given by Brooks, et al [20]. Within NAFNoise, the boundary layer parameters are calculated using XFOIL [21] while the turbulent boundary layer noise is obtained by the method described in [22]. Turbulent inflow noise and trailing edge bluntness noise are neglected. The OASPL results over a frequency range of 1 to $16 \mathrm{kHz}$ are compared in Table 1 for the simulated NACA 0012 and the measured CC noise (where $\Delta=$ CC Noise - NACA 0012 Noise).

The first two cases correspond to $C_{\mu}=0.017$ for the $\mathrm{CC}$ or $\alpha \approx 6.5^{\circ}$ for the NACA 0012 and the latter two $C_{\mu}=0.037$ or $\alpha \approx 13.5^{\circ}$, respectively. All other cases have either been ruled unsuitable within this document or would require angles of attack well above stall. As shown in table 1, CC noise is considerable when compared to a NACA 0012 at the same chord Reynolds number and lift coefficient. At the lower 


\begin{tabular}{cccc}
\hline Case & CC & NACA 0012 & $\Delta \mathrm{dB}$ \\
\hline $\operatorname{Re}_{c}=9.7 e 5, C_{l}=0.72$ & $49 \mathrm{~dB}$ & $46 \mathrm{~dB}$ & $+3 \mathrm{~dB}$ \\
$\operatorname{Re}_{c}=1.3 e 6, C_{l}=0.76$ & $59 \mathrm{~dB}$ & $57 \mathrm{~dB}$ & $+2 \mathrm{~dB}$ \\
$\mathrm{Re}_{c}=6.5 e 5, C_{l}=1.26$ & $44 \mathrm{~dB}$ & $36 \mathrm{~dB}$ & $+8 \mathrm{~dB}$ \\
$\operatorname{Re}_{c}=9.7 e 5, C_{l}=1.32$ & $57 \mathrm{~dB}$ & $46 \mathrm{~dB}$ & $+11 \mathrm{~dB}$ \\
\hline
\end{tabular}

Table 1: OASPL comparison from the CC airfoil experiments to NACA 0012 airfoil estimates from NAFNoise. OASPL computed from 1 to $16 \mathrm{kHz}$.

lift coefficients, the difference is somewhat small; however, as the lift coefficient increases, the CC noise is substantially higher that that generated by the NACA 0012 .

\subsection{Scaling Analysis}

Using the DAMAS results discussed above, a Mach scaling analysis to identify possible acoustic source types is investigated. In order to scale the (y-axis) results, the following equation is employed,

$$
S P L_{S}=10 \log \left[\Delta F\left(\frac{P S D}{P_{r}^{2}}\right)\left(\frac{M_{r}}{M}\right)^{n}\right],
$$

where $P_{r}=20 \mu \mathrm{Pa}, M_{r}=0.1$ and $n$ is the power of Mach number scaling. For proper non-dimensional scaling, the $\mathrm{x}$-axis must also be dimensionless. For scaling versus Helmholtz number $\left(H e=2 \pi F D / c_{0}\right)$, $\Delta F=c_{0} / 2 \pi D$, where $c_{0}$ is the speed of sound, $F$ is frequency, and $D$ is the trailing edge diameter. While scaling against Strouhal number $\left(S t=F D / U_{j}\right), \Delta F=U_{j} / c$, where $c$ is the airfoil chord. It should be noted that the following discussion applies only to the location discussed throughout this document (directly above the CC slot jet). In the event that a highly directive source exists, the scaling may change with observer location.

First, the free-stream Reynolds number is held constant and the jet Mach number is used as a scaling parameter. Figure 28 shows that the spectra in the $R e_{c}=6.5 e 5$ case collapses quite well with jet Mach number to the 6th power for low and intermediate Helmholtz numbers ( $k D$, where $k$ is the wavenumber). This is indicative that a dipole-like source dominates, which can represent either curvature noise or noise generated from turbulence interacting with the slot lip [6]. Based on the scaling Helmholtz range and dominant source locations (discussed above), the low $k D$ noise is likely generated by turbulence from the jet interacting with the curved trailing edge while the middle Helmholtz numbers come from the turbulence in the free-stream interacting with the slot lip [6]. Interestingly, the highest $k D$ noise does not scale well with any power of jet Mach number, suggesting a more complex phenomenon. The same $\left(M^{6}\right)$ scaling is shown in Figure 29 for the $R e_{c}=9.7 e 5$ case. Again, there is a reasonable collapse over low and middle Helmholtz numbers.

The $R e_{c}=0$ case is investigated in Figure 30. For the zero free-stream case a significant collapse continues to be obtained over the lowest Helmholtz numbers; however, unlike the previous cases, the middle $k D$ range do not scale well for all cases. This change can be attributed to the lack of a free-stream; the noise generation mechanism (turbulence interacting with the slot lip) which scaled in the $R e_{c}=6.5 e 5$ cases is no longer present. However, as the jet Reynolds number increases, collapse extends further into the range of moderate Helmholtz numbers, possibly due to increased entrainment acting similar to a free-stream flow. Instead, Figure 31 shows that over the middle range of Strouhal numbers, the spectra collapse reasonably well with jet Mach number to the 5th power. This further indicates a changing dominant acoustic generation mechanism for the middle Helmholtz/Strouhal numbers from the case with no jet to the cases with the jet active.

Finally, the momentum coefficient is held constant (implying a nearly constant jet to free-stream velocity ratio) and scaling is performed versus freestream Mach number. The result is shown for $C_{\mu}=0.017$ in Figure 32. A reasonable collapse is achieved employing Mach scaling to the 7th power. The dominant noise production mechanism is a combination of dipole-like and quadrupole-like sources, suggesting significant noise generation from the interaction between the free-stream and the jet. 


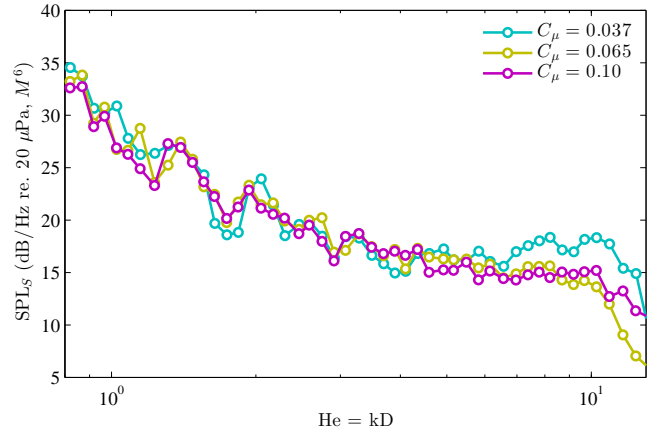

Figure 28: Spectral data from integrated DAMAS at the $1 / 12$ th octave bands centers scaled by jet Mach number to the 6 th power at $R e_{c}=6.5 e 5$.

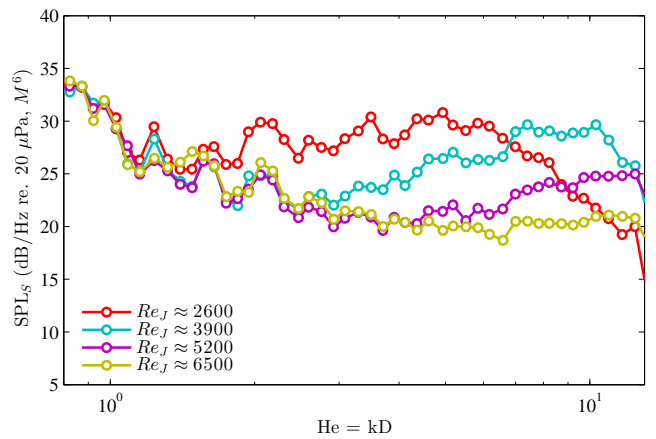

Figure 30: Spectral data from integrated DAMAS at the $1 / 12$ th octave bands centers scaled by jet Mach number to the 6th power at $R e_{c}=0$.

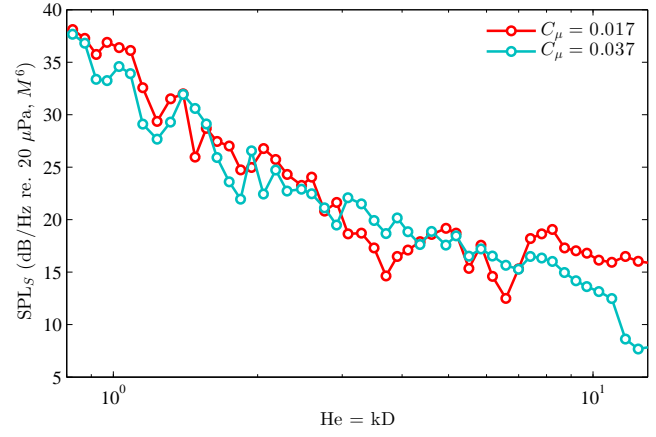

Figure 29: Spectral data from integrated DAMAS at the $1 / 12$ th octave bands centers scaled by jet Mach number to the 6 th power at $R e_{c}=9.7 e 5$.

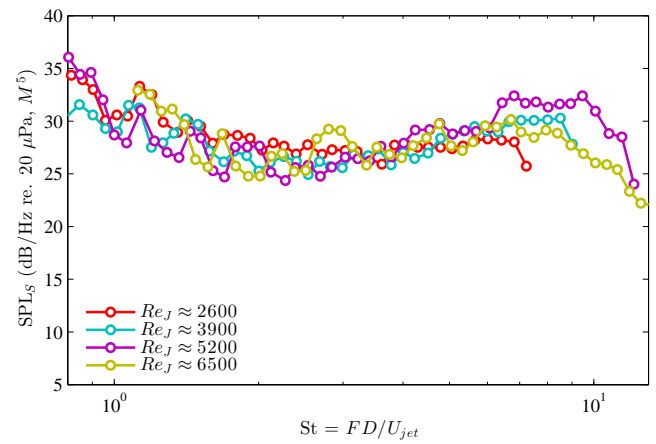

Figure 31: Spectral data from integrated DAMAS at the $1 / 12$ th octave bands centers scaled by Strouhal number and jet Mach number to the 5th power at $R e_{c}=0$.

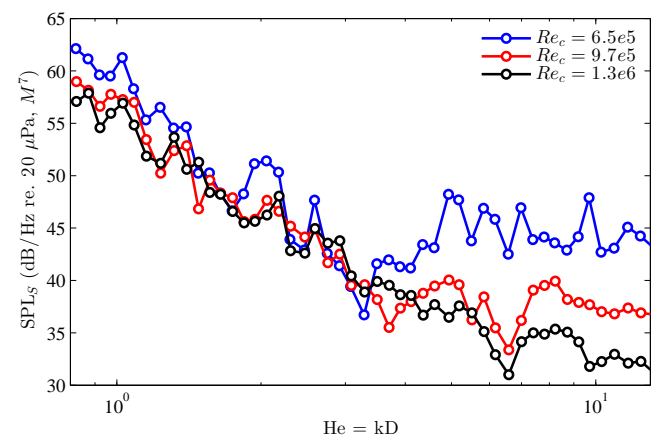

Figure 32: Spectral data from integrated DAMAS at the 1/12th octave bands centers scaled by freestream Mach number to the 7 th power with constant $C_{\mu}=0.017$.

\section{Conclusions}

The present study uses a CC airfoil in an open-jet facility. A 55-microphone phased acoustic array is employed to measure the noise generated from the airfoil. Previous experimental studies regarding the acoustics of circulation control airfoils are limited to primarily single microphone results with some DAS at limited free-stream Reynolds numbers and momentum coefficients. This study investigates the acoustics 
with single microphone and DAS as well as DAMAS beamforming due to significant background noise in the single microphone measurements. Data are acquired at five free-stream Reynolds numbers ranging from 0 to $1.3 \mathrm{e} 6$ and six momentum coefficients from 0 to 0.10 for a fixed slot height of $1 \mathrm{~mm}$.

The single microphone data shows that the spectral noise levels are only significantly above the effective tunnel background noise when the CC jet velocities are large compared to the free-stream. At the lowest non-zero free-stream Reynolds number the CC noise is also shown to be insignificant compared to effective background noise. DAS beamforming is then used to distinguish the noise source of interest from background and model installation noise in the measurement. The background and model installation noise are primarily comprised of tunnel collector noise and CC-sidewall interaction noise while the CC noise is primarily generated by slot-jet interaction. The DAS levels are integrated in space to remove the primary extraneous noise components, which allows the sources of interest to be revealed in the acoustic spectra. In the DAS results, sidelobes are clearly present, especially at high frequencies. DAMAS is then used to remove the effect of the array point-spread-function. Due to the presence of the sidelobes, the DAS also tends to overestimate the levels at middle to high frequencies. While the DAMAS results are well below those of DAS and a single microphone due to the background noise removal, $\mathrm{CC}$ noise is still shown to be well above the noise predicted (by NAFNoise) from a NACA 0012 at the same lift coefficient and free-stream chord Reynolds number.

A scaling analysis is performed on the DAMAS spectra. With a constant free-stream Reynolds number, the acoustic pressure scales well against jet Mach number to the 6th power at low and intermediate Helmholtz numbers. When the free-stream is removed, the jet Mach number to the 6th power scaling remains for low $k D$ but not for the middle. This suggests that Howe's curvature noise remains the dominant source at low Helmholtz numbers for all cases; however, the passive slot noise (which relies on free-stream turbulence interacting with the slot lip) depends on having a significant free-stream to dominate. Finally, reasonable collapse is achieved with a free-stream Mach scaling to the 7th power if the coefficient of momentum is held constant. This suggests that interaction of the free-stream with the jet produces significant acoustic pressure.

\section{Acknowledgments}

The authors would like to acknowledge the financial support for this project provided by the Office of Naval Research grant N00014-12-1-0544, monitored by Dr. Ronald Joslin.

\section{References}

[1] H. Coanda. Propelling device., 1938.

[2] D. A. Wetzel. An experimental investigation of circulation control acoustics. Dissertation, University of Florida, 2011.

[3] R. D. Joslin. Circulation control: Issues for naval applications. In Proceedings of the 2004 NASA/ONR Circulation Control Workshop, 2005.

[4] R. M. Williams and I. C. Cheeseman. Potential acoustic benefits of circulation control rotors. NASA. Langley Research Center Helicopter Acoustics, 2:149-179, 1978.

[5] M. Salikuddin, W. H. Brown, and K. K. Ahuja. Noise from a circulation control wing with upper surface blowing. Journal of Aircraft, 24:55-64, 1987.

[6] M. S. Howe. Noise generated by a Coanda wall jet circulation control device. Journal of Sound and Vibration, 249(4):679$700,2002$.

[7] D. W. Shannon and S. C. Morris. Experimental investigation of slotted circulation control airfoil acoustics. 14th AIAA/CEAS Aeroacoustics Conference (29th AIAA Aeroacoustics Conference), Vancouver, British Columbia, Canada, page $2815,2008$.

[8] S. C. Morris, D. W. Shannon, and J. Silver. Acoustics of a dual-jet circulation control elliptical airfoil. In Proceedings of the 15th AIAA/CEAS Aeroacoustics Conference, Miami, FL, 2009.

[9] D. A. Wetzel, F. Liu, and L. Cattafesta. A theoretical and experimental study of broadband circulation control noise. In 18th AIAA/CEAS Aeroacoustics Conference (33rd AIAA Aeroacoustics Conference) Colorado Springs, CO, volume $2061,2012$.

[10] D. H. Johnson and D. E. Dudgeon. Array Signal Processing: Concepts and Techniques. Prentice Hall, 1993.

[11] T. F. Brooks and W. M. Humphreys. Effect of directional array size on the measurement of airframe noise components. AIA A paper, 1958:1999, 1999.

[12] R. P Dougherty. In Aeroacoustic Measurements, pages 62-97. Springer, 4 edition, 2002. 
[14] E. O. Rogers and M. J. Donnelly. Characteristics of a dual-slotted circulation control wing of low aspect ratio intended for naval hydrodynamic applications. AIAA Paper, 1244, 2004.

[15] K. Pascioni, R. Reger, A. Edstrand, and L. Cattafesta. Characterization of an aeroacoustic wind tunnel facility. In 43rd International Congress of Noise Control Engineering, 2014.

[16] J. R. Underbrink. Practical considerations in focused array design for passive broad-band source mapping applications. Master's Thesis, Pennsylvania State University, 1995.

[17] R. Amiet. Correction of open jet wind tunnel measurements for shear layer refraction. In AIAA Conference on the Exploration of the Outer Planets, volume 1, 1975.

[18] T Yardibi, C. Bahr, N. S. Zawodny, F. Liu, L. N. Cattafesta, and J. Li. Uncertainty analysis of the standard delay-and-sum beamformer and array calibration. Journal of Sound and Vibration, 329:2654-2682, 2010.

454 [19] P. Moriarty. NAFNoise user's guide. In National Wind Technology Center, 2005.

455 [20] T. F. Brooks, D. S. Pope, and M. A. Marcolini. Airfoil self-noise and prediction, volume 1218. National Aeronautics and Space Administration, Office of Management, Scientific and Technical Information Division, 1989.

457 [21] M. Drela. XFOIL: An analysis and design system for low Reynolds number airfoils. In Low Reynolds number aerodynamics, pages 1-12. Springer, 1989.

[22] P. Moriarty and P. Migliore. Semi-empirical aeroacoustic prediction code for wind turbines. In NREL/TP-500-34478, National Wind Technology Center, 2003. 